\section{FACTORES DETERMINANTES DE LA DIVULGACIÓN DE INFORMACIÓN SOBRE EMPRENDIMIENTO POR LOS GOBIERNOS LOCALES ESPAÑOLES}

\author{
Maria Teresa Nevado Gil \\ Patricia Milanés Montero \\ Jorge Manuel Prieto Ballester ${ }^{\text {**** }}$
}

RESUMEN Este trabajo tiene como objetivo examinar los factores que determinan la divulgación de información sobre emprendimiento que realizan los gobiernos locales españoles a través de sus webs. Hoy en día, es evidente la importancia otorgada a los empresarios en las economías locales. Sin embargo, el acceso a la información es asimétrico y la burocracia tiende a limitar la creación y el desarrollo de nuevos negocios. En este escenario, la administración local juega un importante papel en la promoción de un contexto económico adecuado para apoyar el emprendimiento y paliar estos problemas. La metodología utilizada consiste en la aplicación de un modelo de regresión lineal múltiple para medir la influencia de factores poblacionales, socio-económicas y fiscales sobre el indicador de emprendimiento previamente diseñado. Los resultados revelan que la divulgación de información sobre emprendimiento está relacionada con el tamaño del municipio, su capacidad institucional y el nivel de endeudamiento.

Palabras clave: Divulgación de información. Emprendimiento. Factores determinantes. Gobiernos locales. Páginas web.

\footnotetext{
* Doutora pela Universidad de Extremadura, no Programa Oficial de Doctorado en Economía Financiera y Contabilidad, Espanha. Docente e investigadora na Facultad de Empresa, Finanzas y Turismo de la Universidad de Extremadura (Cáceres), Espanha.

E-mail: tnevado@unex.es.

** Doutora pela Universidad de Extremadura, Espanha. Docente e invetigadora na Facultad de Ciencias Económicas y Empresariales da Universidad de Extremadura (Badajoz), Espanha.

E-mail: pmilanes@unex.es.

*** Doutor pela Universidad de Extremadura, no Programa Oficial de Doctorado en Economía Financiera y Contabilidad, Espanha. Docente e investigador na Facultad de Empresa, Finanzas y Turismo de la Universidad de Extremadura (Cáceres), Espanha.

E-mail: jmprieto@unex.es.
}

\section{INTRODUCCIÓN}

a influencia del emprendimiento en el crecimiento económico y la competitividad se ha convertido en un tema clave (VAN STEL et al., 2005; ROMERO; FERNÁNDEZ, 2007; AUDRETSCH, 2009; SULLIVAN; MEEK, 2012). Los estudios contemporáneos revelan una fuerte correlación positiva entre la promoción del espíritu emprendedor y el crecimiento económico, puesto que las actividades empresariales contribuyen al desarrollo de nuevos mercados y mercados ya existentes (MINNITI, 2012). El entorno regional es una variable de contexto importante y no se puede ignorar a la hora de explorar los factores determinantes del emprendimiento y la creación de empresas (STERNBERG, 2009). Sin embargo, la capacidad de la región de beneficiarse de los efectos positivos de la iniciativa empresarial dependerá tanto de sus acuerdos institucionales y su estructura social (BAUMOL, 1990), como de su capacidad para convertir el conocimiento en crecimiento regional a través de su difusión (AUDRETSCH; KEILBACH, 2004).

En este sentido, la administración pública juega un importante papel en el desarrollo económico y regional a través del apoyo y el fomento de la creación de empresas. Además, las nuevas tecnologías, especialmente Internet, facilitan la transmisión de información (DOS SANTOS; BARBOSA, 2011; CARVALHO 
et al., 2017). La tecnología proporciona a las organizaciones una nueva fórmula para interactuar con sus grupos de interés, e, Internet, les ofrece una nueva forma de divulgar la información (BONSÓN; ESCOBAR, 2004; DE MORAES; BARBOSA, 2015). Asimismo, permite que la gestión pública sea más efectiva y eficiente, y mejora la transparencia, la democracia, la participación y la responsabilidad hacia las necesidades sociales (CARVALHO et al., 2017). Las administraciones públicas se esfuerzan por progresar, innovar y transformar la prestación de servicios públicos a través de Internet (WIMMER, 2002). De ahí su interés en saber si las instituciones aprovechan todo el potencial que las Tecnologías de la Información y la Comunicación (TIC) ofrecen para proporcionar información y apoyar el proceso emprendedor a nivel regional.

La divulgación de información a través de sitios web está siendo adoptada cada vez más por las organizaciones y permite una mayor proximidad entre las instituciones y los ciudadanos (JOSEPH; TAPLIN, 2011; MONEVA; MARTIN, 2012; NEVADO et al., 2013). Esta práctica proporciona una mayor y mejor comprensión de las necesidades ciudadanas, debido a la creciente demanda de información generada en los últimos años (RODRÍGUEZ et al., 2010). Muchos estudios han analizado el tipo y el grado de información divulgada por las administraciones a través de sus páginas web. Algunas de estas investigaciones se han centrado en la divulgación de información voluntaria y financiera, sobre sostenibilidad o Responsabilidad Social (RS).

Del mismo modo, tanto la literatura previa como la evidencia empírica disponible revelan que la divulgación de información está asociada a una serie de factores explicativos como el tamaño de la población, la población dependiente, la tasa de desempleo, el nivel educativo, capacidad institucional, el nivel de endeudamiento, la competencia política y la participación electoral, entre otros (CÁRCABA; GARCÍA, 2008; NAVARRO et al., 2010; GUILLAMÓN et al., 2011; ESTELLER-MORÉ; POLO-OTERO, 2012; CRUZ et al., 2012; GARCÍA et al., 2013; CAAMAÑO et al., 2013; ALBALATE, 2013; CUADRADO, 2014). Sin embargo, los estudios sobre la difusión de información relacionada con la capacidad empresarial de las administraciones públicas son todavía escasos. De ahí la originalidad de este estudio, que permitirá, por un lado, obtener evidencia empírica y contribuir a la generación de conocimiento sobre divulgación de información por parte de los gobiernos. Y, por otro lado, promover una actitud positiva hacia el espíritu empresarial y contribuir al desarrollo económico y al aumento del empleo.

En este contexto, el estudio se centra en los gobiernos locales, puesto que representan el primer nivel de participación ciudadana en los asuntos públicos y, por tanto, la divulgación informativa resulta especialmente importante (CAAMAÑO et al., 2013). Al encontrarse las entidades públicas más cercanas al ciudadano, sirven de canal directo e inmediato de participación en los asuntos cotidianos en su localidad (NAVARRO et al., 2010; CUETO et al., 2014) y se sitúan en una posición ventajosa para conocer las demandas informativas de los principales stakeholders, además de favorecer la divulgación (DÍAZ, 2009). Por otro lado, cabe señalar que los compromisos que deben transmitir las entidades locales podrían deberse a la gran variedad de servicios que prestan (NAVARRO et al., 2011), así como a la contraprestación que de esos servicios reciben a través de los impuestos locales (GUILLAMÓN et al., 2011).

Este trabajo tiene como objetivo examinar los factores que afectan a la información sobre emprendimiento suministrada por los gobiernos locales a través de sus páginas web. Para alcanzar este objetivo se ha elegido la Comunidad Autónoma de Extremadura, concretamente, los 213 municipios que conforman la región de Cáceres. La elección de esta Comunidad Autónoma se debe a la elevada tasa de paro que ha registrado en 2017, la más alta del país, con un 28,31\%, según los datos de la Encuesta de Población Activa (INE, 2017). Teniendo esto en cuenta, es relevante estudiar hasta qué punto los municipios pueden contribuir a revertir esta tendencia, a través de la promoción del emprendimiento y los ecosistemas empresariales.

Los resultados de esta investigación podrían proporcionar algunas pistas para la formulación de políticas públicas adaptadas de acuerdo con las economías locales en una perspectiva a largo plazo, puesto que, en el futuro, un número cada vez mayor de personas permanecerá conectado a través de las redes sociales, y las TIC se convertirán en un canal estratégico para comunicarse con las partes interesadas. Este documento está dividido en cuatro partes. En primer lugar, proporciona una revisión de la literatura sobre los 
determinantes de la divulgación de información y presenta las hipótesis de la investigación. En segundo lugar, se muestra el diseño de la investigación llevada a cabo con el propósito de identificar los factores que condicionan la divulgación de información sobre emprendimiento por parte de los gobiernos locales. En tercer lugar, presentamos los resultados y la discusión de los mismos. Terminamos el trabajo analizando sus principales conclusiones, implicaciones y futuras investigaciones.

\section{REVISIÓN DE LITERATURA Y FORMULACIÓN DE HIPÓTESIS}

El emprendimiento, el desarrollo regional y la administración pública desempeñan un papel importante en los territorios y en la interconexión de ciudadanos y empresas para promover el bienestar general. Hoy en día, las TIC son una herramienta común para obtener información, por lo que la divulgación de información a través de los sitios web es una práctica común y permite mejorar la proximidad entre las instituciones y los ciudadanos. Sin embargo, la mayoría de los estudios relacionados con la divulgación de información se han centrado en el sector privado, y es recientemente cuando se ha producido un crecimiento significativo de este tipo de investigación en el sector público (CARVALHO et al., 2017).
Varios estudios han prestado atención a la divulgación de información económica, financiera y no financiera por parte del sector público (LASWAD et al., 2005; CABA et al., 2008), mientras que otros han estudiado la divulgación de información sobre RS en organizaciones públicas (NEVADO et al., 2013; NAVARRO et al., 2015). Sin embargo, aún son escasas las publicaciones centradas en la divulgación de información sobre emprendimiento por parte de las organizaciones públicas. De ahí la importancia del estudio, que tiene como objetivo contribuir a superar esta brecha en la literatura sobre la divulgación de información.

Por lo tanto, la divulgación de información permite a la administración pública comunicarse con sus ciudadanos y minimiza el nivel de asimetría de información entre la administración pública y la sociedad. Este problema de la asimetría de la información entre la administración pública y la sociedad podría limitar la transparencia pública (GUPTA, 2008). Centrándonos en el elemento esencial de nuestra investigación, los gobiernos locales, la revisión de la literatura académica muestra un aumento, en los últimos años, del número de trabajos que analizan los determinantes de la divulgación de información, ya sean económicos, financieros o no financieros, o sobre sostenibilidad o RS. La Tabla 1 muestra una relación de estos estudios.

Tabla 1- Estudios sobre factores determinantes de la divulgación de información

\begin{tabular}{|c|c|c|c|}
\hline Año & Autores & Año & Autores \\
\hline 2004 & Groff y Pitmann & 2011 & Caamaño et al.; Polo-Otero; Guillamón et al. \\
\hline 2005 & Laswad et al. & 2012 & Prado, García y Cuadrado; Cruz et al. \\
\hline 2006 & $\begin{array}{l}\text { Rodríguez, Caba y Hernández; } \\
\text { Alt y Lassen }\end{array}$ & 2013 & $\begin{array}{l}\text { Albalate; Vila; Frías, Marqués y Rodríguez; García } \\
\text { et al. }\end{array}$ \\
\hline 2007 & $\begin{array}{l}\text { Pina et al.; Rodríguez, Caba y } \\
\text { Hernández }\end{array}$ & 2014 & $\begin{array}{l}\text { Joseph, Pilcher y Taplin; Moura, Ribeiro y } \\
\text { Monteiro; Cuadrado }\end{array}$ \\
\hline 2008 & Caba et al.; Gyía y Archidona & 2015 & $\begin{array}{l}\text { Alcaraz, Navarro y Ortiz; Navarro et al.; Mir, } \\
\text { Chatterjee y Taplin; Da Costa, Coutinho y Dos } \\
\text { Santos }\end{array}$ \\
\hline 2009 & Serrano, Rueda y Portillo (a y b) & 2016 & $\begin{array}{l}\text { García, Alonso y Alonso; Mendes, Santos, Ferreira, } \\
\text { Marques, do Carmo y Da Silva; Oates y Moradi- } \\
\text { Motlagh; Brusca, Manes y Aversano; Hashim, } \\
\text { Amran, Nejati y Ismail; Nevado y Gallardo }\end{array}$ \\
\hline 2010 & $\begin{array}{l}\text { Navarro et al.; Cárcaba y García; } \\
\text { Yu }\end{array}$ & 2017 & Carvalho et al. \\
\hline
\end{tabular}

Fuente: nuestra 
De acuerdo con la revisión realizada y, en concreto, con los trabajos más importantes que se ocupan de la identificación de los determinantes de la divulgación de información, hemos considerado apropiado analizar la influencia de las variables que a continuación se detallan.

Tamaño de la población. El tamaño de la empresa es una de las variables más estudiadas en la literatura (SERRANO et al., 2009a; NAVARRO et al., 2010; GARCÍA et al., 2013; NEVADO; GALLARDO, 2016). Los municipios más grandes manejan presupuestos mayores, por lo que, generalmente, tienen más recursos disponibles para destinarlos a incrementar la divulgación de información (GUILLAMÓN et al., 2011). Del mismo modo, Navarro et al. (2010) justifican esta relación argumentando que los municipios de gran población tienen personal mejor cualificado, lo que podría alentar las prácticas de difusión. En general, se puede decir que la literatura previa argumenta la existencia de una relación positiva entre el tamaño del municipio y la divulgación de información (CÁRCABA; GARCÍA, 2008; GARCÍA et al., 2013; NEVADO; GALLARDO, 2016). Tomando como referencia estos argumentos proponemos la siguiente hipótesis:

H1: Existe una relación significativa $y$ positiva entre el tamaño de la población y el nivel de divulgación de información sobre emprendimiento.

Población dependiente. Respecto a esta variable, no existe un amplio consenso, como en el caso del tamaño de la población, sobre el tipo de influencia que ejerce en la divulgación de la información. Autores como Navarro et al. (2011) señalan que cuanto mayor es la población dependiente, mayores son las demandas ciudadanas sobre la información social. Esto podría estar relacionado con el hecho de que la población menor de 19 años y mayor de 65 años requiere un mayor gasto público (NAVARRO et al., 2011). Otros autores, como Nevado y Gallardo (2016), encontraron una influencia negativa de esta variable, es decir, la edad podría estar asociada negativamente con la predisposición a usar internet. Con base en estos argumentos, y teniendo en cuenta que, según datos del padrón municipal de Extremadura, la edad media de los habitantes de la región (43,5 años en 2016) se sitúa por encima de la media nacional, proponemos la siguiente hipótesis:
H2: Existe una relación significativa y negativa entre la población dependiente y el nivel de divulgación de información sobre el emprendimiento.

Deuda municipal. La mayor parte de la literatura muestra que la deuda municipal está relacionada con la transparencia (ALBALATE, 2013; CUADRADO, 2014). Sin embargo, no hay consenso sobre el tipo de influencia que ejerce sobre la divulgación de información. Alt y Lassen (2006) muestran en sus resultados que existe una relación negativa entre el nivel de deuda y la transparencia, puesto que los gobiernos pueden querer ocultar mayores niveles de deuda del público. Sin embargo, otros como Albalate (2013) y Nevado y Gallardo (2016) no encuentran evidencia suficiente que justifique la existencia de una relación significativa entre estas variables. Con base en estos argumentos, proponemos la siguiente hipótesis:

H3: El nivel de endeudamiento de un municipio está relacionado con la divulgación de información sobre emprendimiento.

Capacidad institucional. Otro factor que puede influir en la divulgación de información es el nivel de recursos públicos disponibles (SERRANO et al., 2009a, 2009b; GARCÍA et al., 2013), un factor que representa la capacidad institucional de los gobiernos locales. Laswad et al. (2005) y Serrano et al. (2009a) encontraron una asociación positiva entre la riqueza municipal, medida por el ingreso per cápita, y el nivel de divulgación. Por otro lado, Navarro et al. (2010) apuntan que los municipios con mayores ingresos tienen más argumentos para mejorar sus sistemas de información. Guillamón et al. (2011) y García et al. (2013), que analizaron la capacidad institucional representada por el gasto total, encontraron evidencia de que los gobiernos locales con mayores niveles de gasto revelan más información. Considerando estos argumentos, proponemos la siguiente hipótesis:

H4: Existe una relación significativa y positiva entre la capacidad institucional y el nivel de información divulgada sobre el emprendimiento.

Tasa de desempleo. La tasa de desempleo también se utiliza en la literatura como un posible factor explicativo del nivel de divulgación. El mayor nivel de desempleo implica mayores necesidades sociales $\mathrm{y}$, por tanto, aumenta la presión sobre los gobiernos para que revelen 
información (NAVARRO et al., 2010). Navarro et al. (2011) encontraron una relación significativa y positiva entre la tasa de desempleo y el nivel de información sobre sostenibilidad en 17 gobiernos regionales españoles. Por otro lado, autores como Guillamón et al. (2011), Albalate (2013) y Caamaño et al. (2013) encontraron que los municipios con la tasa de desempleo más alta tienen niveles más bajos de divulgación. Sin embargo, en oposición a estos autores, García et al. (2013) y Nevado y Gallardo (2016) no encontraron ningún tipo de impacto de las tasas de desempleo en la divulgación de información sobre prácticas sostenibles. Con base en estos argumentos, y puesto que la población objeto de este estudio ha sido seleccionada, entre otros aspectos, por su elevada tasa de paro con el propósito de analizar la capacidad de los municipios para revertir esta situación a través de la promoción del emprendimiento mediante el uso de las TICs, se propone la siguiente hipótesis:

H5: La tasa de desempleo está relacionada positivamente con el nivel de divulgación de información sobre emprendimiento.

\section{DISEÑO DE LA INVESTIGACIÓN}

\section{I Selección de la muestra y metodología}

Esta investigación se centra en el estudio de la divulgación de información por parte de la administración local a emprendedores. Como mencionamos anteriormente, la elección de los gobiernos locales se justifica, en primer lugar, porque representan el primer nivel de participación ciudadana en los asuntos públicos (CAAMAÑO et al., 2013). En segundo lugar, debido al papel que juegan en la promoción de la sostenibilidad y el impacto en los ciudadanos por su proximidad y cercanía. Estos se consideran los principales agentes de interés de estas organizaciones (MCTAVISH; PYPPER, 2007; ARAÚJO; TEJEDO, 2016) puesto que, por un lado, contribuyen al sostenimiento del sector público $\mathrm{y}$, por otro lado, son receptores de sus servicios (PINA et al., 2007; GUILLAMÓN et al., 2011; NAVARRO et al., 2016).

Concretamente, se han elegido los gobiernos locales de la región de Cáceres.
Los principales argumentos que respaldan esta decisión son los siguientes: Es una región con gran potencial para proyectos empresariales debido a su buena posición geográfica y accesibilidad. En ella destacan como sectores estratégicos en los que potenciar el emprendimiento, la agricultura, la tecnología, la salud y la calidad de vida, la cultura y el turismo, los servicios, la industria y la energía. Sin embargo, existen desafíos importantes que la región aún enfrenta, como el hecho de que debe orientarse hacia un mayor crecimiento económico y del empleo, a la implementación de un modelo de turismo sostenible y al fortalecimiento de grandes infraestructuras, entre otros.

Además, es una de las regiones menos desarrolladas, con bajas tasas de iniciativa empresarial y una elevada tasa de población envejecida. La tasa de paro registrada en 2017 es la más alta del país, con un $28,31 \%$, según los datos de la Encuesta de Población Activa (INE, 2017). Por otro lado, a pesar de formar parte de una Comunidad Autónoma reconocida como la Región Emprendedora Europea 2017 por construir un ecosistema de apoyo a este sector y a la empresa, Cáceres, aun siendo la segunda región más extensa de España, tan sólo cuenta con dos punto de acompañamiento empresarial para ofrecer orientación en la construcción de proyectos empresariales (información sobre permisos y normativas, opciones de financiación para, ayudas económicas públicas, asesoramiento para expansión a nuevos mercados e internacionalización, gestión de las actividades formativas). Por todo ello, resulta de gran relevancia estudiar hasta qué punto los municipios de la provincia extremeña de Cáceres están desarrollando una adecuada labor de difusión mediante el empleo de las TICs para contribuir a la promoción del emprendimiento y de los ecosistemas empresariales y, por tanto, al crecimiento económico de la región.

La muestra incluye a los 213 municipios de esta región.

El punto de partida de nuestra investigación es la compilación de información relacionada con la divulgación de información sobre emprendimiento, utilizando los datos disponibles en las páginas web de los municipios durante los meses de septiembre y octubre de 2017. La información se obtuvo utilizando la técnica del análisis de contenido 
ampliamente utilizada en investigaciones anteriores (NAVARRO et al., 2011; MONEVA; MARTIN, 2012; NEVADO et al., 2013; BEUREN; ANGONESE, 2015). El instrumento utilizado para la recopilación de información ha sido el cuestionario propuesto por Carvalho et al. (2017), compuesto por un total de 49 indicadores divididos en 5 dimensiones
(Tabla 2). El sistema de puntuación utilizado consistió en asignar a cada indicador el valor 1 si la entidad revela la información relativa a él, y 0 en caso contrario. Este sistema de puntuación ha sido utilizado en estudios empíricos previos de naturaleza similar a este (GANDÍA; ARCHIDONA, 2008; FRÍAS et al., 2013; NEVADO; GALLARDO, 2016).

Tabla 2- Indicadores por dimensiones

\begin{tabular}{llc}
\hline Dimensiones & \multicolumn{1}{c}{ Definición } & $\mathbf{N}^{\mathbf{0}}$ de indicadores \\
\hline Dimensión 1 & Información general & 10 \\
\hline Dimensión 2 & Recursos y apoyo al emprendedor & 12 \\
\hline Dimensión 3 & Emprendimiento activo & 10 \\
\hline Dimensión 4 & Emprendimiento digital & 6 \\
\hline Dimensión 5 & Divulgación y comunicación con el emprendedor & 11 \\
\hline & Total de indicadores & 49 \\
\hline
\end{tabular}

Fuente: Adaptado de Carvalho et al. (2017)

Con la información recopilada, elaboramos un índice de divulgación por municipio siguiendo la metodología utilizada en Carvalho et al. (2017). El uso de índices para medir el nivel de información ha sido utilizado en los estudios de Gandía y Archidona (2008), Navarro et al. (2010 y 2015), Joseph y Taplin (2011), Moneva y Martín (2012) y Beuren y Angonese (2015), entre otros. Una vez calculado el índice de divulgación damos paso al análisis de los factores que condicionan el grado de difusión de información sobre emprendimiento por parte de los gobiernos locales cacereños. Para lograr los objetivos de este estudio y siguiendo a autores como Laswad et al. (2005), Moura et al. (2014) y Alcaraz et al. (2015), entre otros, fundamentamos el análisis en el estudio de los niveles de correlación y en el análisis de regresión, en este caso aplicándose una regresión lineal múltiple. Todas las estimaciones se realizarán utilizando el software SPSS en la versión 20.

\subsection{Variable dependiente: índice de divulgación}

Como se ha señalado anteriormente, el grado de divulgación de información sobre emprendimiento se midió a través de la elaboración de un índice que contiene la puntuación obtenida por cada ayuntamiento después del proceso de revisión y análisis de las páginas web. Previamente a la construcción del índice se calcularon unos índices parciales para cada una de las dimensiones en las que está dividido el cuestionario utilizado. Estos índices parciales se determinan mediante el cociente entre el sumatorio del número de ítems identificados en la web y el número total de ítems que forman cada dimensión.

$$
I D A D_{j}=\frac{\sum_{i=1}^{M}\left(A_{i j}\right)}{M} * 100
$$

Donde, $I D A D=$ Índice de divulgación por ayuntamiento y dimensión; $I D A=$ Índice de divulgación por ayuntamiento; $\mathrm{M}=$ número de ítems que forman cada dimensión; $\mathrm{D}=$ número de dimensiones; = toma el valor de 1 si la característica que define el indicador (i) está presente en el ayuntamiento (j), y 0 en el caso contrario.

El índice de divulgación sobre emprendimiento por municipio () se calcula bajo la siguiente expresión:

$$
I D E_{j}=\sum_{i=1}^{D}\left(I D A D_{j}\right)
$$




\subsection{Variables explicativas}

La selección de los factores determinantes, que pueden influir significativamente en los niveles de información divulgada, se ha realizado después de revisar la literatura previa. Específicamente, los factores identificados como variables independientes del modelo son los incluidos en la Tabla 3:

Tabla 3- Variables explicativas propuestas

Variables/ Hipótesis

\begin{tabular}{cl}
\hline $\begin{array}{c}\text { Tamaño de la población (TP) } \\
\text { H1 }\end{array}$ & $\begin{array}{l}\mathrm{N}^{\mathrm{o}} \text { de habitantes del término } \\
\text { municipal. Datos referidos al último } \\
\text { padrón revisado de } 2016\end{array}$ \\
$\begin{array}{c}\text { Población dependiente (PD) } \\
\text { H2 }\end{array}$ & $\begin{array}{l}\text { Porcentaje de habitantes con edades } \\
<19 \mathrm{y}>65 \text {. Datos referidos al último } \\
\text { padrón revisado de } 2016\end{array}$ \\
\hline $\begin{array}{c}\text { Deuda } \\
\text { Municipal (DM) } \\
\text { H3 }\end{array}$ & $\begin{array}{l}\text { Deuda pública del Municipio año } \\
2016\end{array}$
\end{tabular}

Capacidad institucional (CI) Gasto total per cápita año 2016 $\mathrm{H} 4$

Tasa de desempleo (D) H5
Medidas

Fuente

Instituto Nacional de

Estadística

http://www.ine.es/
Observatorio socioecómico provincial

http:/ / observatorio.dipcaceres.es/
Ministerio de Hacienda y

Administraciones Públicas http:/ / www.minhap.gob.es/

Ministerio de Hacienda y

Administraciones Públicas http://www.minhap.gob.es/

Servicio Público de Empleo

Estatal

http:/ / www.sepe.es/

Fuente: nuestra

\subsection{Especificación del modelo}

Para llevar a cabo el contraste de las hipótesis planteadas en apartados anteriores se emplearon técnicas de regresión multivariante. A través de una regresión lineal múltiple, utilizando el método de Mínimos Cuadrados Ordinarios (MCO), se estimó el siguiente modelo:

$$
I D E_{j}=\beta_{0}+\beta_{1} T P_{j}+\beta_{2} P D_{j}+\beta_{3} D M_{j}+\beta_{4} C I_{j}+\beta_{5} D_{j}+\varepsilon_{j}
$$

Asimismo, se ha incorporado un término error $(\varepsilon)$, que recoge la influencia en el índice de divulgación sobre emprendimiento de los efectos no explicados por las variables independientes.

\section{RESULTADOS Y DISCUSIÓN}

\section{I Análisis descriptivo}

La tabla 4 recoge los estadísticos descriptivos de las variables propuestas (media, desviación estándar, valores máximos y mínimos). Como se puede comprobar, las variables "tamaño poblacional" (TP), "capacidad institucional" (CI) y "deuda municipal" (DM) presentan una notable dispersión que refleja la diversidad existente entre los ayuntamientos del estudio. El resto de las variables no parece tener una gran dispersión. 
Tabla 4- Estadísticos descriptivos

\begin{tabular}{ccrrrr}
\hline & N & Mínimo & \multicolumn{1}{c}{ Máximo } & \multicolumn{1}{c}{ Media } & Desviación \\
\hline $\begin{array}{c}\text { Índice de } \\
\text { divulgación }\end{array}$ & 213 & 0,00 & 62,55 & 12,8843 & 8,31800 \\
\hline $\begin{array}{c}\text { Tamaño de la } \\
\text { población }\end{array}$ & 213 & 51 & 95814 & 1873,27 & 7290,957 \\
\hline $\begin{array}{c}\text { Capacidad } \\
\text { institucional }\end{array}$ & 213 & 0,00 & 65487414,20 & 1574810,2389 & 5267822,47675 \\
\hline Deuda municipal & 213 & 0,00 & 39407136,82 & 471557,5056 & 3074528,11703 \\
\hline $\begin{array}{c}\text { Población } \\
\text { dependiente }\end{array}$ & 213 & 25,49 & 57,48 & 43,2441 & 5,11152 \\
\hline Tasa de desempleo & 213 & 0,0672 & 0,4918 & 0,233969 & 0,0656811 \\
\hline
\end{tabular}

Fuente: nuestra

\subsection{Análisis univariante}

Con el fin de comprobar si existe correlación entre las variables del modelo se aplica el coeficiente de correlación de Pearson (Tabla 5), que mide el grado de asociación existente generalmente entre dos variables aleatorias. La matriz de correlaciones muestra que la mayoría de las variables independientes están correlacionadas de forma moderada o baja, a excepción de algunas variables que presentan una correlación fuerte. Sin embargo, ninguna de estas correlaciones alcanza el valor crítico de 0,80 de acuerdo con Cooper y Schindler (2008) y Naser y Hassan (2013), por lo que no comprometen la aplicación del modelo y se mantienen todas las variables.

Tabla 5- Matriz de correlaciones de Pearson de las variables incluidas en el estudio

\begin{tabular}{cccccc}
\hline & $\mathrm{TP}$ & $\mathrm{PD}$ & $\mathrm{DM}$ & $\mathrm{CI}$ & $\mathrm{D}$ \\
\hline $\mathrm{TP}$ & 1 & & & & \\
\hline $\mathrm{PD}$ & 0,061 & 1 & & & \\
\hline $\mathrm{DM}$ & $-0,118$ &,$- 140^{*}$ & 1 & & \\
\hline $\mathrm{CI}$ & $-0,193^{* *}$ & $-0,200^{* *}$ & $0,791^{* *}$ & 1 & \\
\hline $\mathrm{D}$ & 0,062 & $-0,102$ & 0,032 & 0,012 & 1 \\
\hline
\end{tabular}

Fuente: nuestra

Note. $\left.{ }^{* *}\right)$ correlaciones significativas al nivel 1\%; $\left(^{*}\right)$ correlaciones significativas al nivel $5 \%$ (bilateral).

\subsection{Análisis multivariante}

Los resultados obtenidos se presentan en la Tabla 6. El muestra que el modelo tiene una capacidad explicativa del $30,7 \%$, porcentaje considerablemente alto al tratarse de datos de corte transversal (GUJARATI, 2009). Por otro lado, en la Tabla 6 se muestra también el F-statistic $(18,315)$, que mide si las variables independientes incluidas en nuestro modelo, de manera conjunta, influyen en el índice de divulgación sobre emprendimiento. Si observamos el valor del F-sig $(0,0000)$, inferior a 0,01 , podemos concluir que existe al menos un $99 \%$ de confianza de que el modelo propuesto explique nuestra variable dependiente.

Tabla 6- Resultados de la regresión lineal múltiple

\begin{tabular}{|c|c|c|c|}
\hline Variables & Coeficiente & $\mathbf{t}$ & Sig. \\
\hline (Constante) & 15,947 & 3,361 & 0,001 \\
\hline Tamaño población (TP) & 399,378 & 2,154 & $0,032^{* *}$ \\
\hline Población dependiente (PD) & $-11,035$ & $-1,141$ & 0,255 \\
\hline Deuda municipal (DM) & 7,745E-007 & 3,019 & $0,003^{* * *}$ \\
\hline Capacidad institucional (CI) & $3,784 \mathrm{E}-007$ & 2,474 & $0,014^{* *}$ \\
\hline Tasa de desempleo (D) & 7,287 & 0,986 & 0,325 \\
\hline $\begin{array}{l}\text { F-statistic } \\
\text { F-sig } \\
\mathrm{R}^{2}\end{array}$ & $\begin{array}{r}18,315 \\
0,0000 \\
0,307\end{array}$ & & \\
\hline
\end{tabular}

Fuente: nuestra

Variable dependiente (IDE). ${ }^{* * *}$ ) indica significatividad al nivel $1 \%$; $\left.{ }^{* *}\right)$ indica significatividad al nivel $5 \%$. 
En cuanto a la significatividad individual de las variables independientes, como se puede observar en la Tabla 6, la variable representativa del tamaño de la población (TP) se muestra significativa al $95 \%$ de confianza (t-Statistic $=3,361 ; \quad$ Probabilidad $=0,032) \quad y$ está asociada positivamente con la variable dependiente (IDE), tal y como muestra el coeficiente asociado $(=399,378)$. Confirmamos, por tanto, nuestra hipótesis $\mathrm{H} 1$, lo que indica que cuanto mayor sea el tamaño de los ayuntamientos cacereños, mayor será su índice de divulgación sobre emprendimiento. Estos resultados son consistentes con los de otros autores como Ryan et al. (2002), Larrán y Giner (2002), Cárcaba y García (2008) y García et al. (2013).

Respecto a la deuda municipal (DM) se ha obtenido una influencia significativa al $95 \%$ de confianza (t-Statistic=3,019; Probabilidad=0,003). Si observamos el signo del coeficiente $(=7,745 \mathrm{E}-$ 007) comprobamos que está relacionado positivamente con el nivel de divulgación de información sobre emprendimiento. Confirmamos, por tanto, la hipótesis H3 que nos indica que los ayuntamientos más endeudados presentan mayores niveles de divulgación. Estos resultados son contradictorios con los de Alt y Lassen (2006), que muestran la existencia de una relación negativa entre el nivel de deuda y la transparencia, puesto que los gobiernos pueden querer ocultar mayores niveles de deuda del público.

Referente a la capacidad institucional (CI), comprobamos a través de los estadísticos (t-Statistic $=2,474 ; \quad$ Probabilidad $=0,014$ ), que es significativa individualmente a un $95 \%$ de confianza y también está asociada positivamente con la variable dependiente (ID) $(=3,784 \mathrm{E}-$ 007), confirmando así nuestra hipótesis H2. Los resultados obtenidos coinciden con los alcanzados en trabajos previos (LASWAD et al., 2005; GANDÍA; ARCHIDONA, 2008; GUILLAMÓN et al., 2011), donde se han encontrado mayores niveles de divulgación de información en municipios que cuentan con mayores recursos.

El resto de las variables (población dependiente y tasa de desempleo) no son significativas en nuestro modelo, por lo que no tienen efecto sobre la variable dependiente. Esto nos lleva al rechazo de las hipótesis H4 y H5. Según los resultados obtenidos para nuestra muestra, una creciente población dependiente o una mayor o menor tasa de desempleo no explican el comportamiento de los partidos gobernantes a la hora de divulgar información sobre emprendimiento.

\section{CONCLUSIONES, IMPLICACIO- NES Y FUTURAS LÍNEAS DE IN- VESTIGACIÓN}

Este estudio analiza la divulgación de información sobre emprendimiento en el contexto de entidades locales a nivel regional, específicamente en la región de Cáceres, identificando los factores que influyen en el volumen de información divulgada. Concretamente, los resultados obtenidos se dividen en dos áreas. Por un lado, mediante los resultados obtenidos se evidencia la existencia de una serie de factores que explican el comportamiento de los ayuntamientos en relación con la divulgación de información sobre emprendimiento, que son el tamaño de la población, la capacidad institucional del municipio y su nivel de endeudamiento.

Por otro lado, y contrariamente a nuestras expectativas, la población dependiente y la tasa de desempleo no son factores explicativos del nivel de divulgación. Esto se debe a que sólo existe un grupo de ayuntamientos en nuestra muestra comprometidos con el apoyo al emprendimiento a través de las TICs. Con estos resultados, los municipios ya pueden enfocar sus prácticas y políticas de divulgación de información sobre emprendimiento en respuesta a los factores que influyen en el nivel de divulgación

Podemos concluir, por tanto, que aquellos municipios cacereños de mayor tamaño, con mayor capacidad institucional, y mayor nivel de endeudamiento divulgan más información sobre emprendimiento a través de sus páginas web. Al mismo tiempo, parece que ni la población dependiente ni la tasa de desempleo de un municipio motivan a los gobiernos de la región de Cáceres a divulgar este tipo de información.

En cuanto a las implicaciones, podemos enfatizar que, en el ámbito académico, nuestra investigación contribuye a la literatura existente con una mayor explicación de lo que está sucediendo en relación con la divulgación 
de información sobre emprendimiento en el sector público. Así, con base en los resultados obtenidos, tenemos la intención de completar la literatura, así como alentar a los municipios a mejorar la divulgación de información sobre emprendimiento a través de sus páginas web. Asimismo, los resultados de esta investigación podrían proporcionar algunas pistas, tanto a corto como a largo plazo, sobre la formulación de políticas públicas adaptadas a las economías locales y los municipios.

Somos conscientes de que nuestra investigación no está exenta de aspectos que limitan los resultados obtenidos. Por un lado, los efectos de la región y el país deben ser considerados. En este caso, todos los municipios analizados pertenecen a la región de Cáceres (España), sin embargo, la consideración de entidades pertenecientes a otras regiones $\mathrm{e}$ incluso a otros países concluiría con resultados más profundos.
En consecuencia, y en términos de futuras investigaciones, sería conveniente abordar el estudio con una muestra más amplia que permita realizar análisis comparativos a nivel de países, así como llevar a cabo un estudio longitudinal que permita el análisis de la evolución de la información divulgada en esta materia.

\section{Agradecimientos:}

Este trabajo fue apoyado por el Grupo Banco Santander y la Diputación de Cáceres, y respaldado por el Plan de Iniciación a la Investigación, Desarrollo Tecnológico e Innovación 2017 de la Universidad de Extremadura (Acción VII).

Artigo recebido em I2/02/20 I8 e aceito para publicação em 21/02/20।8

\section{FACTORS DETERMINING THE DISCLOSURE OF INFORMATION ON ENTREPRENEURSHIP BY THE SPANISH LOCAL GOVERNMENTS}

ABSTRACT The objective of this work is to examine the factors that determine the disclosure of information on entrepreneurship carried out by Spanish local governments through their web pages. Nowadays, the importance given to entrepreneurs in local economies is evident. However, in many situations, the access to information is asymmetric and the bureaucracy tends to limit the creation and the development of new businesses. In this scenario, the local administration plays an important role in promoting an adequate economic context to support entrepreneurship, mainly to alleviate the problems arising from asymmetric information. The methodology used consists of the application of a multiple linear regression model to measure the influence of population, socio-economic and fiscal factors on the entrepreneurial indicator designed. The results show that the dissemination of information on entrepreneurship is related to the size of the municipality, its institutional capacity and the level of indebtedness.

Keywords: Disclosure. Entrepreneurship. Determinants. Local government. Websites.

\section{REFERENCIAS}

ALBALATE DEL SOL, D. The institutional, economic y social determinants of local government transparency. Journal of Economic Policy Reform, v. 16, n. 1, p. 90-107, 2013.
ALCARAZ, F. J; NAVARRO, A; ORTIZ, D. 2015. Factors determining online sustainability reporting by local governments. International Review of Administrative Sciences, v. 81, n. 1, p. 79-109, 2015.

ALT, J. E; LASSEN, D. D. Fiscal transparency, political parties, $\mathrm{y}$ debt in OECD countries. 
European Economic Review, v. 50, n. 6, p. 14031439, 2006.

ARAÚJO, J. F. F. E. D; TEJEDO, F. Local government transparency index: determinants of municipalities' rankings. International Journal of Public Sector Management, v. 29, n. 4, p. 327347, 2016.

AUDRETSCH, D. B; KEILBACH, M. Entrepreneurship y regional growth: An evolutionary interpretation. Journal of Evolutionary Economics, v.14, n. 5, p. 605-616, 2004.

AUDRETSCH, D. B. Capital emprendedor y crecimiento económico. Investigaciones Regionales, v. 15, p. 27-45, 2009.

BAUMOL, W. J. Entrepreneurship: Productive, unproductive y destructive. Journal of Political Economy, v. 98, n. 5, p. 892-921, 1990.

BEUREN, I. M; Angonese, R. Instruments for determining the disclosure index of accounting information. Revista eletronica de estrategia e negocios-reen, v. 8, n. 1, p. 120-144, 2015.

BONSÓN, E; ESCOBAR, T. La difusión voluntaria de información financiera en Internet. Un análisis comparativo entre Estados Unidos, Europa del Este y la Unión Europea. Spanish Journal of Finance y Accounting/Revista Española de Financiación y Contabilidad, v. 33 n. 123, p. 1063-1101, 2004.

CAAMAÑO, A. J; LAGO PEÑAS, S; REYES SANTIAS, F; SANTIAGO BOUBETA, A. Budget transparency in local governments: an empirical analysis. Local Government Studies, v. 39, v. 2, p. 182-207, 2013.

CABA, P. C; RODRÍGUEZ, B. M; LÓPEZ, H. A. $\mathrm{M}$. E-Government process y incentives for online public financial information. Online Information Review, v. 32, n. 3, p. 379-400, 2008.

CÁRCABA， A; GARCÍA， J. Determinantes de la divulgación de información contable a través de Internet por parte de los gobiernos locales. Revista Española de Financiación y Contabilidad, v. 37, n. 137, p. 63-84, 2008.

CARVALHO, L; GALLARDO, V. D; NEVADO, G. M. T. Local municipalities's involvement in promoting entrepreneurship: An analysis of web pages orientation to the entrepreneurs in Portuguese municipalities. In: Hybook of Research on Entrepreneurial Ecosystems y Social Dynamics in a Globalized World, IGI, ISBN13: 9781522535256, 2017.

COOPER, D. R; SCHINDLER, P. S. Business research methods (10th ed.). Boston: McGrawHill Irwin, 2008.

CRUZ, C. F; FERREIRA, A. C. D. S; SILVA, L. M. D; MACEDO, M. Á. D. S. Transparency of the municipal public management: a study from the homepages of the large Brazilian municipalities. Revista de Administração Pública, v. 46, n. 1, p. 153-176, 2012.

CUADRADO, B. B. The impact of functional decentralization $\mathrm{y}$ externalization on local government transparency. Government Information Quarterly, v. 31, n. 2, p. 265-277, 2014.

CUETO, C. C; DE LA CUESTA, G. M; MONEVA, A. J. M. La oferta informativa sobre Responsabilidad Social Corporativa en las administraciones locales españolas. Revista Prisma Social, Revista de Ciencias Sociales, n. 12, p. 646-687, 2014.

DÍAZ, C. V. Transparencia de la información económico-financiera a través del e-Gobierno o Gobierno Electrónico: Caso Español. Perspectivas, n. 24, p. 59-90, 2009. 
DE MORAES, L. B; BARBOSA, R. R. Cultura informacional: uma proposta de modelo com foco organizacional. Informação \& Sociedade, v. 25, n. 3, p. 131-146, 2015.

DOS SANTOS, A. P; BARBOSA, R. R. Desafios da mobilidade corporativa para a Gestão da Informação e do Conhecimento. Informação \& Sociedade, v. 21, n.2, p. 49-62, 2011.

ESTELLER-MORÉ, A; POLO-OTERO, J. Fiscal Transparency: (Why) does your local government respond? Public Management Review, v. 14, n. 8, p. 1153-1173, 2012.

FRÍAS, A. J. V; MARQUES, M. D. C; RODRÍGUEZ, A. L. Divulgación de información sostenible: ¿se adapta a las expectativas de la sociedad? Spanish Accounting Review/ Revista de contabilidad, v. 16, n. 2, p. 147-158, 2013.

GYIA, J. L; ARCHIDONA, M. C. Determinants of web site information by Spanish city councils. Online information review, v. 32, n. 1, p. 35-57, 2008.

GARCÍA, S.I.M; FRÍAS, A. J. V; RODRÍGUEZ D. L. Determinants of corporate social disclosure in Spanish Local governments. Journal of Cleaner Production, v. 39, p. 60-72, 2013.

GANDÍA, J. L; ARCHIDONA, M. C. Determinants of web site information by Spanish city councils. Online Information Review, v. 32, n. 1, p. 35-57, 2008.

GUILLAMÓN, M. D; BASTIDA, F; BENITO, B. The determinants of local government's financial transparency. Local Government Studies, v. 37, n. 4, p. 391-406, 2011.

GUJARATI, D. N. Basic Econometrics. New York: Tata McGraw-Hill Education, 2009.
GUPTA, A. Transparency Under Scrutiny: Information Disclosure in Global Environmental Governance. Global environmental politics, v. 8, n. 2, p. 1-7, 2008.

INSTITUTO NACIONAL DE ESTADÍSTICA. Encuesta de población activa, Madrid, 2017.

JOSEPH, C; TAPLIN, R. The measurement of sustainability disclosure: Abundance versus occurrence. Accounting Forum, v. 35, n. 1, p. 1931, 2011.

JOSEPH, C; PILCHER, R; TAPLIN, R. Malaysian Local Government internet sustainability reporting. Pacific Accounting Review, v. 26, n. 1/2, p. 75-93, 2014.

LARRÁN, M; GINER, B. The Use of the Internet for Corporate Reporting by Spanish Companies. The International Journal of Digital Accounting Research, v. 2, n. 3, p. 53-82, 2002.

LASWAD, F; FISHER, R; OYELERE, P. Determinants of voluntary Internet financial reporting by local government authorities. Journal of Accounting y Public Policy, v. 25, p. 101-121, 2005.

MCTAVISH, D; PYPER, R. Monitoring the Public Appointments process in the UK. Public Management Review, v. 9, n. 1, p. 145-153, 2007.

MINNITI, M. El emprendimiento y el crecimiento económico de las naciones. Economía industrial, n. 383, p. 23-30, 2012.

MONEVA, J; MARTIN, E. Universidad y Desarrollo sostenible: Análisis de la rendición de cuentas de las universidades públicas desde un enfoque de responsabilidad social. Revista Iberoamericana de Contabilidad de Gestión, v. 10, n. 19, p. 1-18, 2012. 
MOURA, A; RIBEIRO, V; MONTEIRO, S. Divulgação de Informação na internet sobre Responsabilidade Social-Evidência Empírica nos Municípios Portugueses. In: XXIV JORNADAS LUSO-ESPANHOLAS DE GESTÃO CIENTÍFICA, 2014.

NASER, K; HASSAN, Y. Determinants of corporate social responsibility reporting: Evidence from an emerging economy. Journal of Contemporary Issues in Business Research, v. 2, n. 3, p. 56-74, 2013.

NAVARRO, G. A; ALCARAZ, F. J; Ortiz, D. La divulgación de información sobre responsabilidad corporativa en administraciones públicas: un estudio empírico en gobiernos locales. Revista Española de Contabilidad, RCSAC, v.13, n. 2, p. 285-314, 2010.

NAVARRO, G. A; ORTIZ, D; ALCARAZ, F. J; ZAFRA, J. L. La divulgación de información sobre sostenibilidad en los gobiernos regionales y sus factores influyentes: el caso de España." In: XVI CONGRESO AECA, 2011.

NAVARRO, G. A; TIRADO, V. P; RUIZ, L. M; DE LOS RÍOS, B. A. Divulgación de información sobre responsabilidad social de los gobiernos locales europeos: El caso de los países nórdicos. Gestión y política pública, v. 24, n. 1, p. 229-270, 2015.

NAVARRO, G. A; ALCARAZ, F. J; ORTIZ, D. Online dissemination of information on sustainability in regional governments. Effects of technological factors. Government Information Quarterly, v. 33, n. 1, p. 53-66, 2016.

NEVADO, G. M. T; GALLARDO, V. D; SÁNCHEZ, H. M. I. La administración local y su implicación en la creación de una cultura socialmente responsable. Revista Prisma Social, Revista de Ciencias Sociales, v. 10, p. 64-118, 2013.
NEVADO, G. M. T; GALLARDO, V. D. Información sobre Responsabilidad Social contenida en las páginas web de los ayuntamientos. Estudio en la región del Alentejo. Revista española de Documentación Científica, v. 39, n. 4, p. 150, 2016.

PINA, V; TORRES, L; ACERETE, B. Are ICTs promoting governments accountability? A comparative analysis of e-governance developments in 19 OECD countries. Critical Perspectives on Accounting, v. 18, n. 5, p. 583602, 2007.

RYAN, C; STANLEY, T; NELSON, M. Accountability Disclosures by Queensland Local Government Councils: 1997-1999. Financial Accountability and Management, v. 183, p. 261289, 2002.

RODRÍGUEZ, D. L; GALLEGO, A. I; GARCÍA, S. I. M. Determinantes de la divulgación voluntaria de información estratégica en internet: un estudio de las empresas cotizadas. Revista Europea de Dirección y Economía de la Empresa, v. 19, n. 1, p. 9-26, 2010.

ROMERO, L. I; FERNÁNDEZ, S. J. Un análisis de la heterogeneidad empresarial en la Unión Europea. Implicaciones para la política europea de fomento empresarial. Ekonomiaz, v. 66 n. 3, p. 350-371, 2007.

SERRANO, C. C; RUEDA, T. M; PORTILLO, T. P. Factors influencing e-disclosure in local public administrations. Environment y Planning C-Government y Policy, v. 27, n. 2, p. 355-378, 2009a.

SERRANO, C. C; RUEDA, T. M; PORTILLO, T. P. Determinants of e-government extension. Online Information Review, v. 33, n. 3, p. 476498, 2009b. 
STERNBERG, R. Regional Dimensions of Entrepreneurship. University of Hannover, Germany, 2009.

SULLIVAN, D. M; MEEK, W. R. Gender y entrepreneurship: A review y process model. Journal of Managerial Psychology, v. 27, n. 5, p. 428-458, 2012.
VAN STEL, A; CARREE, M; THURIK, R. The effect of entrepreneurial activity on national economic growth. Small business economics, v. 24, n. 3, p. 311-321, 2005.

WIMMER, M. A. Integrated service modelling for online one-stop government. Electronic Markets, v. 12, n. 3, p. 149-156, 2002. 